\title{
A supplementary grading scale combining lesion-to-eloquence distance for predicting surgical outcomes of patients with brain arteriovenous malformations
}

\author{
Yuming Jiao, MD, ${ }^{1-4}$ Fuxin Lin, MD, ${ }^{1-4}$ Jun Wu, MD, ${ }^{1-4} \mathrm{Hao} \mathrm{Li}^{1,-4}$ Lijun Wang, MD, ${ }^{6}$ Zhen Jin, MD, ${ }^{5}$ \\ Shuo Wang, MD, ${ }^{1-4}$ and Yong Cao, MD ${ }^{1-4}$
}

\begin{abstract}
${ }^{1}$ Department of Neurosurgery, Beijing Tiantan Hospital, Capital Medical University; ${ }^{2}$ China National Clinical Research Center for Neurological Diseases; ${ }^{3}$ Center of Stroke, Beijing Institute for Brain Disorders; ' ${ }^{4}$ Beijing Key Laboratory of Translational Medicine for Cerebrovascular Disease; ${ }^{5}$ Medical Imaging Center, 306th Hospital of the People's Liberation Army, Beijing; and ${ }^{6}$ Department of Neurosurgery, Hongqi Hospital, Mudanjiang Medical University, Mudanjiang, Heilongjiang, People's Republic of China
\end{abstract}

OBJECTIVE Case selection for the surgical treatment of brain arteriovenous malformations (BAVMs) remains challenging. This study aimed to construct a predictive grading system combining lesion-to-eloquence distance (LED) for selecting patients with BAVMs for surgery.

METHODS Between September 2012 and September 2015, the authors retrospectively studied 201 consecutive patients with BAVMs. All patients had undergone preoperative functional MRI and diffusion tensor imaging (DTI), followed by resection. Both angioarchitectural factors and LED were analyzed with respect to the change between preoperative and final postoperative modified Rankin Scale (mRS) scores. LED refers to the distance between the lesion and the nearest eloquent area (eloquent cortex or eloquent fiber tracts) measured on preoperative fMRI and DTI. Based on logistic regression analysis, the authors constructed 3 new grading systems. The HDVL grading system includes the independent predictors of $\mathrm{mRS}$ change (hemorrhagic presentation, diffuseness, deep venous drainage, and LED). Full Score combines the variables in the Spetzler-Martin (S-M) grading system (nidus size, eloquence of adjacent brain, and venous drainage) and the HDVL. For the third grading system, the fS-M grading system, the authors added information regarding eloquent fiber tracts to the S-M grading system. The area under the receiver operating characteristic (ROC) curves was compared with those of the S-M grading system and the supplementary S-M grading system of Lawton et al. RESULTS LED was significantly correlated with a change in $\mathrm{mRS}$ score $(p<0.001)$. An LED of $4.95 \mathrm{~mm}$ was the cutoff point for the worsened $\mathrm{mRS}$ score. Hemorrhagic presentation, diffuseness, deep venous drainage, and LED were independent predictors of a change in mRS score. Predictive accuracy was highest for the HDVL grading system (area under the ROC curve 0.82 ), followed by the Full Score grading system (0.80), the fS-M grading system (0.79), the supplementary S-M grading system (0.76), and least for the S-M grading system (0.71). Predictive accuracy of the HDVL grading system was significantly better than that of the Spetzler-Martin grade $(p=0.040)$.

CONCLUSIONS LED was a significant predictor for the preoperative risk evaluation for surgery. The HDVL system was a good predictor of neurological outcomes after BAVM surgery. Adding the consideration of the involvement of eloquent fiber tracts to preoperative evaluation can effectively improve its predictive accuracy.

https://thejns.org/doi/abs/10.3171/2016.10.JNS161415

KEY WORDS brain arteriovenous malformation; modified Rankin Scale; prediction grading systems; lesion-toeloquence distance; Spetzler-Martin grade; HDVL grading system; vascular disorders

\footnotetext{
ABBREVIATIONS AF = arcuate fasciculus; $A U C=$ area under the ROC curve; $B A V M=$ brain arteriovenous malformation; $B O L D=$ blood oxygen level-dependent; $C S T=$ cortical spinal tract; DSA = digital subtraction angiography; DTI = diffusion tensor imaging; DW = diffusion weighted; EPI = echo-planar imaging; fMRI = functional MRI; fS-M = S-M grading system with the addition of eloquent fiber tracts; Full Score = all variables in HDVL and S-M grading systems; HDVL = a grading system including hemorrhage, nidus diffuseness, deep venous drainage, and LED; LED = lesion-to-eloquence distance; $\mathrm{mRS}=$ modified Rankin Scale; ROC = receiver operating characteristic; $\mathrm{ROI}=$ region of interest; $\mathrm{S}-\mathrm{M}=$ Spetzler-Martin; TOF = time-of-flight.
}

SUBMITTED June 3, 2016. ACCEPTED October 21, 2016.

INCLUDE WHEN CITING Published online March 31, 2017; DOI: 10.3171/2016.10.JNS161415. 
$\mathrm{C}$ AREFUL case selection is necessary to minimize postoperative complications and neurological deficits after the resection of brain arteriovenous malformations (BAVMs). When surgery is considered, grading schemes are used to aid in predicting the associated risks. The prediction model most frequently used is the Spetzler-Martin (S-M) grading system ${ }^{22}$ in which ordinal scores are assigned to 3 variables: AVM size, eloquence of location, and pattern of venous drainage. The S-M grading system facilitates stratification of BAVM characteristics among patients and the comparison of clinical outcomes among institutions. To increase the predictive accuracy of the S-M grading system, previous studies have supplemented it with other variables. ${ }^{15}$ However, there are still shortcomings that restrict the predictive value of either the S-M grading system or the supplementary grading system. First, the definition of eloquence of location is inadequate. In the S-M grading system, the sensorimotor, language, and visual cortices are considered. However, the grading system does not include white matter eloquent fiber tracts, such as the subcortical cortical spinal tract (CST), optic radiation, and arcuate fasciculus (AF) with respect to these functions. ${ }^{22}$ Second, the definition of eloquence is not clear. There are no definite criteria establishing an acceptable distance between the nidus and eloquent brain. Moreover, there is a lack of quantitative variables to describe the relationship between nidus and eloquence. The somewhat arbitrary definition of eloquence has led to highly variable coding when data from different AVM centers have been compared, thereby suggesting a low interrater reliability of this criterion. ${ }^{6,12}$ Higher predictive accuracy may be achieved if these defects are remedied.

In recent years, the noninvasive study techniques of functional MRI (fMRI) and diffusion tensor imaging (DTI) have become important functional neuroimaging techniques in clinical practice..$^{1,418}$ By detecting the change in signal of a complex physiological phenomenon called the blood oxygen level-dependent (BOLD) effect, fMRI helps us more precisely localize neurological function and allows us to determine a patient's eloquent cortex. ${ }^{5}$ With DTI tractography, the spatial relationship between passing fibers and the BAVM nidus can be easily visualized. With the help of these 2 techniques, distances between nidus and eloquence, both eloquent cortex and fiber tracts (lesion-to-eloquence distance [LED]), can be measured. These modalities have served as reliable tools for preoperative lesion localization and patient-specific trajectory planning for BAVM.$^{8,16}$ In previous studies, we demonstrated that the LED measured using fMRI and DTI data was a significant predictor of surgical outcomes for BAVM located in eloquent motor area or the occipital lobe. ${ }^{17,23}$ However, as far as we know, there are still no prediction models for surgical outcomes of BAVM that include the LED. Whether adding LED information to a previous grading system can be used to build a supplementary grading system that would better predict neurological outcome remains unclear.

To better understand the predictive role of LED and build a better prediction model that includes LED, we retrospectively analyzed a consecutive surgical series of 201 patients to determine the factors that significantly corre- lated with surgical outcome and built different predictive models to compare their predictive accuracy.

\section{Methods \\ Study Population}

We retrospectively reviewed our BAVM database of a prospective randomized controlled clinical trial (ClinicalTrials.gov registration no. NCT0175821125 of patients who had undergone microsurgical resection of their BAVM between September 2012 and September 2015. All patients had undergone fMRI and DTI. A total of 201 patients were enrolled. This study adhered to good clinical practice and ethical principles described in the Declaration of Helsinki, and it was approved by the IRB of Beijing Tiantan Hospital Affiliated with Capital Medical University. Written informed consent was obtained from all participants or their legally authorized representative.

\section{Neuroimaging}

Computed tomography, MRI, and digital subtraction angiography (DSA) studies were obtained in all patients. Functional MRI and DTI were performed using a 3.0T MR system (SIEMENS Trio) within 1 week before surgery. The sagittal T1-weighted anatomical image acquired was a gradient-echo sequence (TR $2300 \mathrm{msec}$, TE $2.98 \mathrm{msec}$, slice thickness $1 \mathrm{~mm}$, slices 176, FOV 256 $\mathrm{mm}$, flip angle $9^{\circ}$, matrix $64 \times 64$, voxel size $1 \times 1 \times 1$ $\mathrm{mm}^{3}$, bandwidth 240). The BOLD fMRI sequences were performed using standard echo-planar imaging (EPI): TR $3000 \mathrm{msec}$, TE $30 \mathrm{msec}$, matrix $64 \times 64$, axial slices 30 including all cerebral areas, isotropic resolution 3 $\mathrm{mm}$. Repetitive finger-to-thumb opposition movements or flexion-extension movements of the foot were used as the motor stimulation paradigms, with 64 repetitions and a 24-second task and 24-second control state alternately. A silent reading task was performed as the language stimulation paradigm. Patients were asked to read Chinese characters presented on the screen silently and to understand the meaning of each word or sentence. The stimulus was a paragraph composed of Chinese characters, which were shown through a light-emitting diode projector system. During the paradigm, two 30-second trials were performed and interleaved with three 20 -second conditions. The maps of neural activity within the visual cortex were generated with a task of "black-white checkerboard." The DTI was performed using the diffusion-weighted (DW)-EPI technique: TR $6100 \mathrm{msec}$, TE $93 \mathrm{msec}$, slice thickness $3 \mathrm{~mm}$, slices 45, FOV $230 \times 230$ $\mathrm{mm}^{2}$, matrix $128 \times 128$ with a motion-probing gradient in 30 orientations. Axial time-of-flight (TOF) MR angiography was performed using a 3D TOF gradient-echo acquisition sequence (TR $22 \mathrm{msec}$, TE $3.86 \mathrm{msec}$, slice thickness $1 \mathrm{~mm}$, slices $36 \times 4$, FOV $220 \times 220 \mathrm{~mm}^{2}$, flip angle $120^{\circ}$, matrix $\left.512 \times 512\right) .{ }^{17}$

\section{Neuroimaging Data Analyses}

Generated image sets were processed using the iPlan 3.0 workstation (Brainlab). All image sets were automatically coregistered with each other and fused to the anatomical images by an automatic rigid registration. 
Eloquent cortices included all the cortices described in the S-M grading system (the sensorimotor, language, and visual cortices). ${ }^{22}$ A significance threshold of $p<0.001$ $(T=6.5)$ was considered for identification of activated clusters. Two experienced neurosurgeons (J.W. and F.L.), on consensus, documented the anatomical locations of the activation and peak activity point for each paradigm. They were blinded to the patient outcomes. White matter eloquent fiber tracts, including the subcortical CST, optic radiation and $\mathrm{AF}$ with respect to the function described in the S-M grading system, were considered to be eloquent. ${ }^{22}$ We used the inferior portion of the pons and precentral gyrus as the regions of interest (ROIs) to track the CST. ${ }^{24}$ The posterior parietal area of the superior longitudinal fascicle and the posterior temporal lobe were selected to track the AF.13 The lateral geniculate nucleus and calcarine cortex were used as the ROIs to track the optic radiation..$^{20}$ Tractography was performed with fiber propagation stopped at a fractional anisotropy threshold of less than 0.20 . A minimum fiber length of $70 \mathrm{~mm}$ was selected. The anatomical locations of the ROIs and detailed information about the tracked eloquent fiber tracts were documented by the 2 neurosurgeons (J.W. and F.L.) with consensus. All the processed data sets were incorporated into the neuronavigation platform for intraoperative neuronavigation. In this study, the mean cost of fMRI and DTI scans was 2000 RMB (renminbi) per patient.

\section{Surgery}

The treatment strategy for BAVM was selected based on patient condition, perceived surgical risks, and patient requirements. Conservative treatment was selected for patients without a history of prior hemorrhage, with symptoms well controlled by medications, or with an S-M grade of VI.22 Only patients with intractable seizures, severe headache, low S-M grade, progressive neurological deficits, and previous hemorrhages were considered for surgery. Microsurgical resection of BAVM was performed by an experienced senior physician (S.W.). The BAVM was surgically resected with special attention to preserve white matter tracts using a neuronavigation system. Intraoperative ultrasonography and indocyanine fluorescence angiography were used in all cases to discern the margin and feeding arteries of BAVM. CT scanning was performed immediately whenever there was neurological deterioration to rule out intracranial hemorrhage. DSA was also performed to validate radical obliteration 5 days after surgery.

\section{Study Variables and Definition}

Patient demographics, including age and sex, were collected. Angioarchitectural variables for each BAVM (size, diffuseness, deep draining veins, presence of deep perforating arterial feeders, hemorrhagic presentation, eloquence of adjacent brain, and S-M grade), were determined from findings on preoperative angiograms, traditional MRI scans, and TOF images. Diffuseness was determined from preoperative angiograms, with TOF images used to identify intervening brain parenchyma within the nidus. Hemorrhagic presentation was defined as radio-
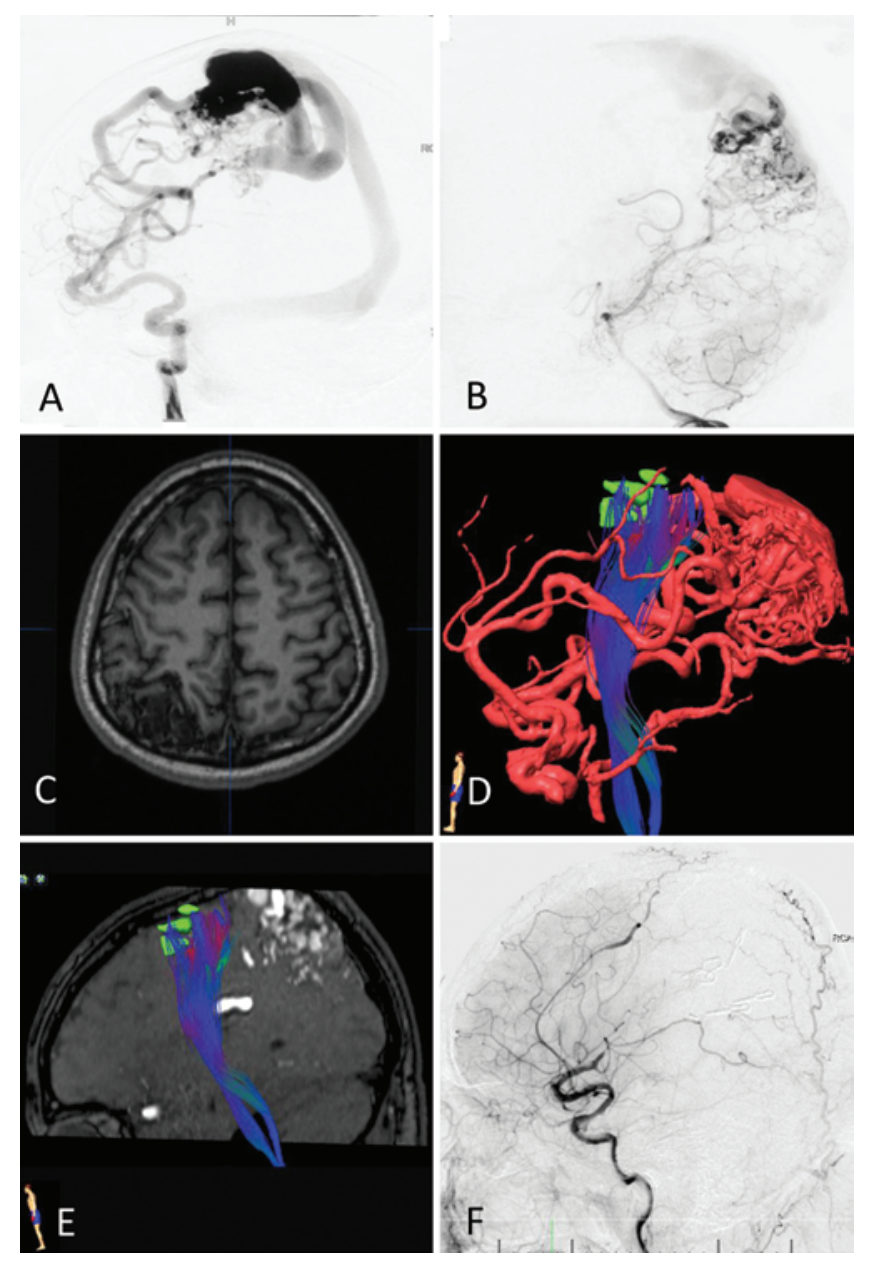

FIG. 1. This 23-year-old man presented with an unruptured AVM in the right parietal lobe. A and B: Angiograms (right internal carotid artery injection $[A]$ and vertebral artery injection $[B]$ ) demonstrating superficial venous drainage and confirming a diffuse border inferiorly and posteriorly. C: Axial T1-weighted MR image showing that the AVM is just posterior to the somatosensory strip. D: Preoperative fMRI study showing that the nidus was not close to the eloquent cortex (green area). E: Preoperative DTI tractography image showing that the nidus was close to the left CST and the LED was $3.8 \mathrm{~mm}$. Therefore, based on the findings in panels $D$ and $E$, this patient had an S-M Grade II AVM (S2V0E0, where $S$ denotes size; $V$, venous drainage; and $E$, eloquent adjacent brain) and an HDVL Grade 5 (H1D1V0L3, where H denotes hemorrhage; $D$, diffuseness; $V$, deep venous drainage; and L, LED) (mismatched grades). F: Postoperative angiogram showing the radical resection. The patient had new muscle strength deficits postoperatively. The muscle strength of the left limbs was Grade 3 at 7 days after surgery and recovered to Grade 4 at last follow-up. The supplementary grade was more predictive of his final outcome. Figure is available in color online only.

graphic evidence of hemorrhage on CT or MRI, regardless of signs or symptoms. The functional variable, LED, refers to distance between lesion and the nearest eloquent cortex or eloquent fiber tracts. The eloquent cortex was identified using fMRI (Figs. 1 and 2), and the eloquent fiber tracts were visualized using DTI tractography (Fig. 3). The quantification of the LED was performed by an experienced neurosurgeon (H.L.) and an experienced neuroradiologist (Z.J.) with consensus. They were blinded to the patient out- 

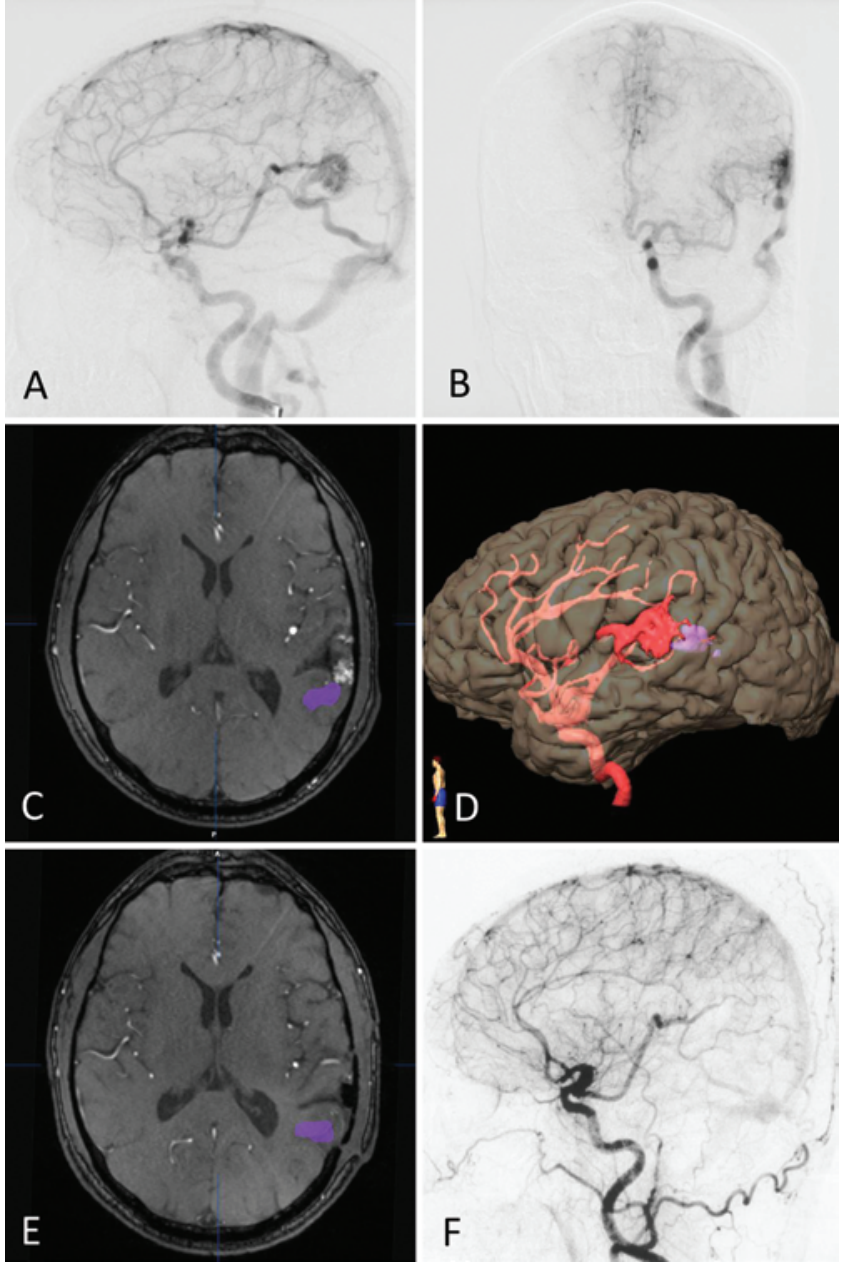

FIG. 2. This 45-year-old man presented with a temporal lobe hemorrhage as seen on axial TOF images. A and B: Angiograms (left internal carotid artery injection, lateral $[\mathrm{A}]$ and anteroposterior $[\mathrm{B}]$ views) demonstrating a small, compact nidus with superficial venous drainage. $C$ and D: Preoperative axial (C) and 3D reconstructed (D) fMR images showing that the nidus was close to Wernicke's area. Therefore, this patient had an S-M Grade II AVM (S1V0E1) and an HDVL Grade 3 (HODOVOL3)

(matched grades). E: Postoperative fMR image showing the intact Wernicke's area. F: Postoperative angiogram showing the radical resection. The patient recovered fully. Figure is available in color online only.

comes. Sections for measurement were manually selected where the activated area or the tracked fiber appeared to be nearest to the margin of the nidus on the TOF images fused with fMRI and DTI tractography images.

We used the modified Rankin Scale (mRS) to assess outcomes, as previously described.$^{15}$ Briefly, a nurse clinician who was supervised by a neurologist evaluated mRS scores at presentation, preoperatively, 7 days postoperatively, and at the final follow-up. The mean duration of the last follow-up was 14.2 months (range 3-34 months). Patient outcomes were assessed based on the difference between the preoperative and final postoperative mRS scores (final mRS score - preoperative mRS score). Good outcome was defined as a final mRS score of $0-2$, and poor outcome was considered as a final $\mathrm{mRS}$ score greater
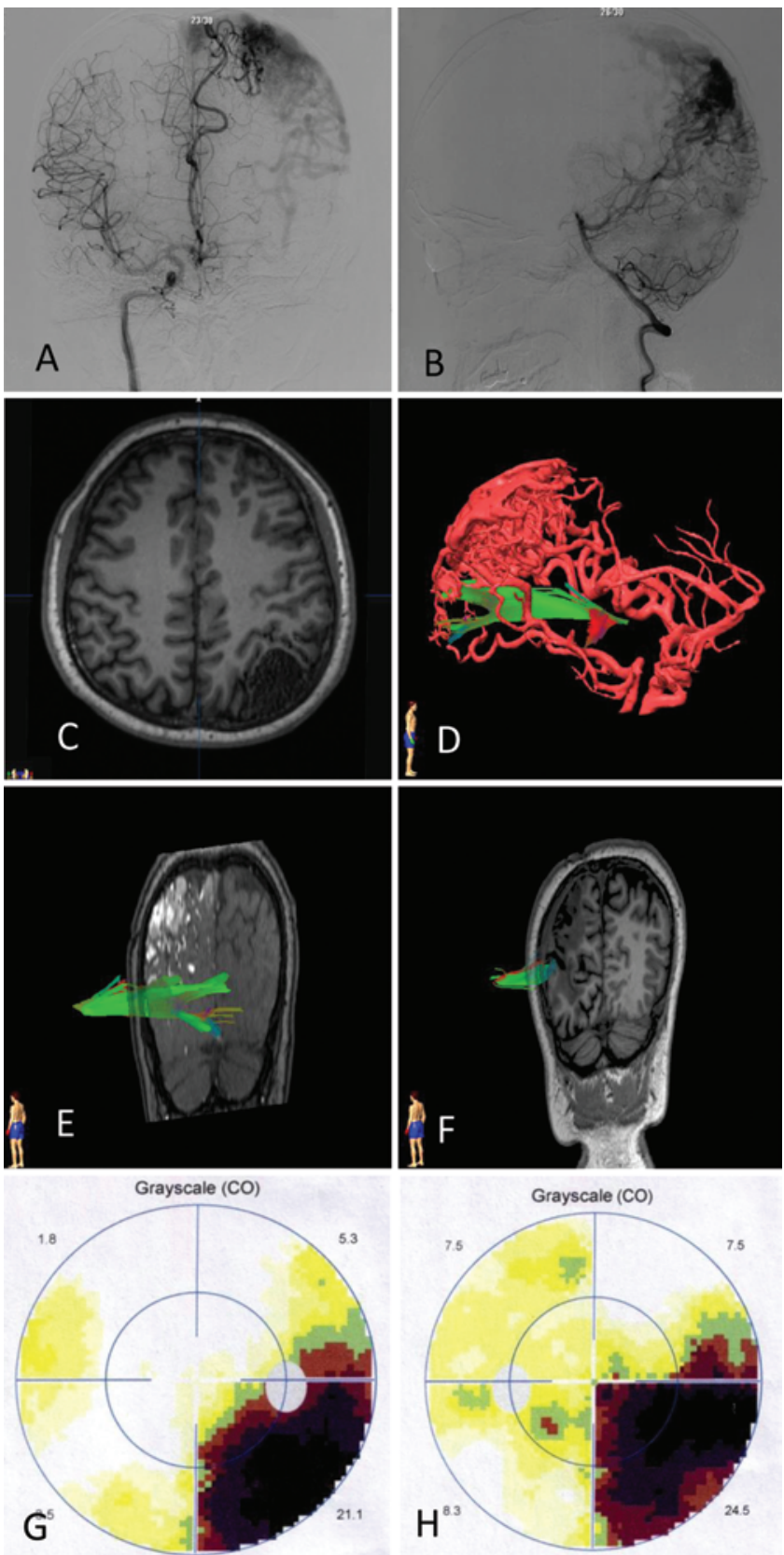

FIG. 3. This 33-year-old man presented with an unruptured AVM in the left parietal lobe. A and B: Angiograms (left internal carotid artery injection $[A]$ and vertebral artery injection $[B])$ demonstrating superficial venous drainage and confirming a diffuse border medially and posteriorly. C: Axial T-1 weighted image showing the AVM just posterior to the somatosensory strip. D and E: Preoperative DTI tractography images showing that the nidus involved the left optic radiation and the LED was 0 . Therefore, this patient had an S-M Grade II AVM (S2V0E0, where $S$ denotes size; $V$, venous drainage; and $E$, eloquent adjacent brain) and an HDVL Grade 5 (H1D1V0L3, where H denotes hemorrhage; $D$, diffuseness; $V$, deep venous drainage; and L, LED) (mismatched grades). F: Postoperative DTI tractography image showing that the optic radiation was ruptured. $\mathbf{G}$ and $\mathrm{H}$ : The patient had new visual field deficits postoperatively in the right $(\mathrm{G})$ and left $(\mathrm{H})$ eyes. The supplementary grade was more predictive of his final outcome. $\mathrm{CO}=$ co-occurrence. 
than 2. A change in mRS score of less than or equal to 0 indicated that the patient's condition had improved or remained the same, whereas a change in $\mathrm{mRS}$ score of greater than 0 indicated that the patient's condition had deteriorated. Two experienced neurosurgeons (Y.J. and F.L.) collected the clinical information from the prospectively collected database and electronic medical records system.

\section{Statistical Analysis}

Statistical analysis was performed using the statistical package SPSS (version 20.0.0, IBM). A chi-square test or Fisher's exact test was used for dichotomized variables. Continuous variables were compared using the independent samples t-test. Univariate and multivariate logistic regression analyses were used to test the association of each predictor. Patients with a change in $\mathrm{mRS}$ score of $1-6$ indicated deterioration, and a change $\leq 0$ indicated that there was improvement or no change in the patient's condition. Different multivariable logistic models were constructed to test the association of combined predictor variables with the change in $\mathrm{mRS}$ score. We developed our 3 scoring systems based on the predictors by using beta coefficients from the multivariable logistic regression models to weight the clinical and BAVM characteristic values for each patient. ROC analyses were performed for each grading system, and the area under the ROC curves (AUC) were compared for accuracy in predicting change in $\mathrm{mRS}$ score among these 4 models (i.e., S-M, HDVL, fS-M, and Full Score).

\section{Model Construction}

According to logistic regression, 5 different multivariable logistic models were constructed to test the association of combined predictor variables with the change in mRS score (Table 1): the HDVL grade included the 4 independent predictors according to the multivariable logistic regression analyses (hemorrhage presentation, nidus diffuseness, deep venous drainage, and LED). The S-M grading system is the classic S-M grading system comprising BAVM size, adjacent eloquent cortex, and nidus deep venous drainage. The supplementary S-M grading system, the grading scale proposed by Lawton et al., ${ }^{15}$ adds patient age, nidus diffuseness, and hemorrhagic presentation to the S-M grading system. The $\mathrm{fS}-\mathrm{M}$ grade refers to the S-M grading system plus information regarding the eloquent fiber tracts. The Full Score includes all variables of the S-M grading system and HDVL grading system. Refer to Table 1 for the scores. Multivariable logistic regression analysis was performed. We used the beta coefficients from the multivariable logistic regression models to weight the clinical and BAVM characteristic values for the 3 new grading systems.

Because we used all the data to build our prediction models, which could result in overly optimistic predictions, we performed a 10 -fold cross validation. The AUC was then estimated using data from the 10 validation sets.

\section{Results}

\section{Patient and BAVM Characteristics}

Demographic and BAVM characteristics of the patient
TABLE 1. Grading systems of BAVMs derived from the cohort

\begin{tabular}{|c|c|c|c|c|}
\hline Variable & Full Score & S-M & HDVL & fS-M \\
\hline \multicolumn{5}{|l|}{ Size $(\mathrm{cm})$} \\
\hline$<3$ & 1 & 1 & & 1 \\
\hline $3-6$ & 2 & 2 & & 2 \\
\hline$>6$ & 3 & 3 & & 3 \\
\hline \multicolumn{5}{|l|}{ S-M eloquence* } \\
\hline No & 0 & 0 & & \\
\hline Yes & 1 & 1 & & \\
\hline \multicolumn{5}{|l|}{ Eloquence $†$} \\
\hline No & & & & 0 \\
\hline Yes & & & & 1 \\
\hline \multicolumn{5}{|c|}{ Deep venous drainage } \\
\hline No & 0 & 0 & 0 & 0 \\
\hline Yes & 1 & 1 & 1 & 1 \\
\hline \multicolumn{5}{|c|}{ Preop hemorrhage } \\
\hline No & 1 & & 1 & \\
\hline Yes & 0 & & 0 & \\
\hline \multicolumn{5}{|l|}{ Diffuseness } \\
\hline No & 0 & & 0 & \\
\hline Yes & 1 & & 1 & \\
\hline \multicolumn{5}{|l|}{ LED (mm) } \\
\hline $0-5$ & 3 & & 3 & \\
\hline $5-10$ & 2 & & 2 & \\
\hline$>10$ & 1 & & 1 & \\
\hline
\end{tabular}

cohort are presented in Tables 2 and 3. Of the 201 patients, $12(6.0 \%)$ underwent embolization, radiosurgery, or both prior to microsurgical resection in select cases. Good outcomes after BAVM resection were observed in 190 of the 201 patients $(94.5 \%$, mRS Scores $0-2)$. Based on changes in $\mathrm{mRS}$ score, the conditions remained the same or improved in 152 patients $(75.6 \%)$ and worsened in 49 patients (24.4\%); no patient died. Compared with patients whose conditions improved or remained unchanged, those whose conditions had worsened had BAVMs that were larger $(p<0.001)$, diffuse $(p<0.001)$, with deep venous drainage $(p=0.001)$, eloquent adjacent cortex $(\mathrm{p}=0.042)$, nonhemorrhagic presentation $(\mathrm{p}=$ $0.003)$, shorter LED ( $<$ 0.001), and higher S-M grade $(\mathrm{p}<0.001)$. There was no significant difference between patient age $(\mathrm{p}=0.684)$, sex $(\mathrm{p}=0.313)$, nidus side $(\mathrm{p}=$ $0.055)$, deep perforating artery supply $(p=0.078)$, and change in mRS score.

\section{Logistic Regression Analysis}

Univariable logistic regression analysis identified nidus size $(\mathrm{p}<0.001)$, nonhemorrhagic presentation $(\mathrm{p}=0.006)$, diffuse nidus ( $<0.001)$, eloquence $(p=0.044)$, and deep venous drainage $(p=0.002)$ as significant predictors of a worsened mRS score. However, there was no significant relationship between patient age $(\mathrm{p}=0.682)$, sex $(\mathrm{p}=$ 
TABLE 2. Demographic, angioarchitectural, and functional characteristics

\begin{tabular}{|c|c|}
\hline Variables & Value \\
\hline Total patients & 201 \\
\hline \multicolumn{2}{|l|}{ Demographic variables } \\
\hline Mean age in yrs & $28.8 \pm 12.9$ \\
\hline \multicolumn{2}{|l|}{ Sex } \\
\hline Male & $127(63.2)$ \\
\hline Female & $74(36.8)$ \\
\hline \multicolumn{2}{|l|}{ Side } \\
\hline Left & $106(52.7)$ \\
\hline Right & $95(47.3)$ \\
\hline \multicolumn{2}{|l|}{ Angioarchitecture } \\
\hline Mean size in $\mathrm{mm}$ & $37.9 \pm 14.5$ \\
\hline \multicolumn{2}{|l|}{ Hemorrhage } \\
\hline Yes & $48(23.9)$ \\
\hline No & $153(76.1)$ \\
\hline \multicolumn{2}{|l|}{ Deep venous drainage } \\
\hline Yes & $23(11.4)$ \\
\hline No & $178(88.6)$ \\
\hline \multicolumn{2}{|l|}{ Diffuse nidus } \\
\hline Yes & $66(32.8)$ \\
\hline No & $135(67.2)$ \\
\hline \multicolumn{2}{|l|}{ Arterial perforator supply } \\
\hline Yes & $58(28.9)$ \\
\hline No & $143(71.1)$ \\
\hline \multicolumn{2}{|l|}{ Functional variables } \\
\hline \multicolumn{2}{|l|}{ S-M eloquence } \\
\hline Yes & $74(36.8)$ \\
\hline No & $127(63.2)$ \\
\hline Mean LED in mm & $8.3 \pm 10.6$ \\
\hline Mean S-M grade & $2.3 \pm 0.9$ \\
\hline Preop ND & $34(16.9)$ \\
\hline Mean preop mRS score & $1.1 \pm 0.6$ \\
\hline Mean FU in mos (range) & $14.2 \pm 8.6(3-34)$ \\
\hline Postop infection & $12(6.0)$ \\
\hline Short-term (1 wk) ND & $106(52.7)$ \\
\hline Mean short-term mRS score & $1.6 \pm 1.0$ \\
\hline Long-term ND & $72(35.8)$ \\
\hline Mean long-term mRS score & $0.9 \pm 1.0$ \\
\hline
\end{tabular}

$\mathrm{FU}=$ follow-up; $\mathrm{ND}=$ neurological deficits.

Values indicate the number of patients (\%) unless otherwise indicated. Mean values are presented as the mean \pm SD.

$0.314)$, nidus side ( $p=0.056$ ), deep perforating artery supply $(\mathrm{p}=0.080)$ and worsened mRS score (Table 4). Multivariable logistic regression analyses showed that deep venous drainage $(p=0.029)$, nidus diffuseness $(p=0.005)$, nonhemorrhagic presentation $(p=0.014)$, and shorter LED $(p<0.001)$ were independent risk factors for a worsened mRS score.

Multivariable logistic regression analyses using the
TABLE 3. Patient and BAVM characteristics by change in preoperative and postoperative $\mathrm{mRS}$ score

\begin{tabular}{|c|c|c|c|}
\hline \multirow[b]{2}{*}{ Variable } & \multicolumn{2}{|c|}{ Outcome mRS Score } & \multirow[b]{2}{*}{$p$ Value } \\
\hline & $0-2$ & $\geq 2$ & \\
\hline No. of patients & $152(75.6)$ & $49(24.4)$ & \\
\hline \multicolumn{4}{|l|}{ Demographic variables } \\
\hline Mean age in yrs & $28.6 \pm 13.3$ & $29.4 \pm 11.4$ & 0.684 \\
\hline Sex & & & 0.313 \\
\hline Male & $99(78.0)$ & $28(22.0)$ & \\
\hline Female & $53(71.6)$ & $21(28.4)$ & \\
\hline Side & & & 0.055 \\
\hline Left & $86(81.1)$ & $20(18.9)$ & \\
\hline Right & $66(69.5)$ & $29(30.5)$ & \\
\hline \multicolumn{4}{|l|}{ Angioarchitectural variables } \\
\hline Size in $\mathrm{mm}$ & $35.6 \pm 13.3$ & $45.0 \pm 16.1$ & $<0.001$ \\
\hline Hemorrhage & & & 0.003 \\
\hline Yes & $44(91.7)$ & $4(8.3)$ & \\
\hline No & $108(70.6)$ & $45(29.4)$ & \\
\hline Deep venous drainage & & & 0.001 \\
\hline Yes & $11(47.8)$ & $12(52.2)$ & \\
\hline No & $141(79.2)$ & $37(20.8)$ & \\
\hline Diffuse nidus & & & $<0.001$ \\
\hline Yes & $38(57.6)$ & $28(42.4)$ & \\
\hline No & $114(84.4)$ & $21(15.6)$ & \\
\hline Arterial perforator supply & & & 0.078 \\
\hline Yes & $39(67.2)$ & $19(32.8)$ & \\
\hline No & $113(79)$ & $30(21.0)$ & \\
\hline \multicolumn{4}{|l|}{ Functional variables } \\
\hline S-M eloquence & & & 0.042 \\
\hline Yes & $50(67.6)$ & $24(32.4)$ & \\
\hline No & $102(80.3)$ & $25(19.7)$ & \\
\hline Mean LED in mm & $10.2 \pm 11.4$ & $2.6 \pm 4.2$ & $<0.001$ \\
\hline Mean S-M grade & $2.1 \pm 0.8$ & $2.8 \pm 0.9$ & $<0.001$ \\
\hline Mean preop mRS score & $1.2 \pm 0.6$ & $0.9 \pm 0.4$ & 0.006 \\
\hline Postop infection & & & 0.690 \\
\hline Yes & $8(66.7)$ & $4(33.3)$ & \\
\hline No & $144(76.2)$ & $45(23.8)$ & \\
\hline Mean FU in mos & $14.6 \pm 8.9$ & $13.1 \pm 7.7$ & 0.283 \\
\hline
\end{tabular}

Full Score model identified preoperative nonhemorrhagic presentation $(\mathrm{p}=0.024)$, deep drainage $(\mathrm{p}=0.027)$, and shorter LED $(p=0.001)$ as independent and significant predictors of worsened $\mathrm{mRS}$ score. In the S-M grading system, BAVM size $(\mathrm{p}<0.001)$ and deep venous drainage $(\mathrm{p}=0.001)$ were significant predictors of worsened $\mathrm{mRS}$ score. In the HDVL model, nonhemorrhagic presentation $(p=0.013)$, diffuseness $(p=0.005)$, deep venous drainage $(p=0.033)$, and LED ( $p<0.001)$ were all independent predictors of worsened outcome (Tables 5 and 6). 
TABLE 4. Univariable logistic regression analysis comparing patients who experienced neurological improvement or remained unchanged with those who experienced deterioration

\begin{tabular}{lrrr}
\hline \multicolumn{1}{c}{ Variable } & OR & $95 \% \mathrm{Cl}$ & $\mathrm{p}$ Value \\
\hline Age & 1.00 & $0.98-1.03$ & 0.682 \\
\hline Sex & 1.40 & $0.73-2.70$ & 0.314 \\
\hline Size & 1.05 & $1.02-1.07$ & $<0.001$ \\
\hline Side & 1.89 & $0.98-3.63$ & 0.056 \\
\hline Deep venous drainage & 4.16 & $1.70-10.17$ & 0.002 \\
\hline Arterial perforator supply & 1.84 & $0.93-3.62$ & 0.080 \\
\hline S-M eloquence & 1.96 & $1.02-3.77$ & 0.044 \\
\hline Hemorrhage & 0.22 & $0.07-0.64$ & 0.006 \\
\hline Diffuseness & 4.00 & $2.04-7.85$ & $<0.001$ \\
\hline S-M grade & 2.58 & $1.70-3.91$ & $<0.001$ \\
\hline LED & 0.88 & $0.82-0.94$ & $<0.001$ \\
\hline FU in mos & 0.98 & $0.94-1.02$ & 0.282 \\
\hline Bolface type indicates stistica
\end{tabular}

Boldface type indicates statistical significance.

\section{Measures of Discrimination}

The discrimination of the model was determined using ROC curves to test the model's ability to correctly identify patients whose conditions will worsen (mRS score change $>0$ ). The predictive accuracy of the HDVL grade was the highest (AUC 0.82, 95\% CI 0.76-0.88), which was significantly better than that of the S-M grade (AUC $0.71,95 \%$ CI $0.63-0.79 ; \mathrm{p}=0.040$ ). The predictive accuracy of the HDVL grading system was also better than the Full Score (AUC $0.80,95 \%$ CI $0.74-0.87$ ) and the supplementary S-M grading system (AUC $0.76,95 \%$ CI $0.69-0.84$ ). The AUC of the fS-M was 0.79 (95\% CI 0.72-0.86). The S-M grading system had the lowest predictive accuracy (Table 5 and Fig. 4). Neurological outcomes by Full Score grade, S-M grade, fS-M grade, and HDVL grade are shown in Table 7. A greater percentage of patients had worsened neurological outcomes with increasing HDVL grade, with stratification into a low-risk group (0\%-11.8\%, Grades
$1-3)$ and high-risk groups (31.5\%- 62.5\%, Grades 4-6) (Tables 7 and 8).

To evaluate whether the predictive accuracy of our supplementary grade was overly optimistic, we performed a 10 -fold cross-validation of the data. The 10-fold cross-validation resulted in estimates similar to the HDVL grading system with an AUC of 0.80 compared with 0.82 , suggesting that the model was not overly optimistic.

\section{Discussion}

In this study, our data demonstrated that the LED determined from fMRI and DTI tractography findings was a significant predictor for the preoperative risk evaluation for surgery. The HDVL grading system (nonhemorrhagic presentation, deep venous drainage, nidus diffuseness, and LED) had a high predictive value of neurological outcomes after BAVM surgery. In addition, adding information regarding the eloquent fiber tracts to the S-M grading system can effectively improve its predictive accuracy.

In this study, our data showed that nonhemorrhagic presentation, deep venous drainage, nidus diffuseness, and LED were independent predictors for surgical outcomes. Consistent with our data, several studies have reported that nidus diffuseness, nonhemorrhagic presentation, and deep venous drainage were significantly correlated with surgical morbidity. ${ }^{10,15,21}$ As previously described, compact BAVMs have distinct dissection planes with clear separation between the nidus and brain tissue, whereas diffuse BAVMs have obscure planes that can draw the dissection too close to the nidus, resulting in hemorrhagic complications, or can force the surgeon to resect interspersed brain, which might injure the functional cortex or fiber tracts. ${ }^{7}$ In addition, previous hemorrhage may facilitate surgery. Hematomas help separate BAVM from adjacent brain; thus, evacuation of a hematoma creates a working space around the BAVM that can minimize transgression of normal brain or provide access to a deep nidus that might otherwise have been unreachable. ${ }^{15}$ Moreover, deep drainage veins are friable, resist bipolar coagulation, and have the dangerous propensity to

TABLE 5. Multivariable logistic regression analysis for Full Score model, S-M grade, and supplementary S-M grade

\begin{tabular}{|c|c|c|c|c|c|c|c|c|c|}
\hline \multirow[b]{2}{*}{ Variables } & \multicolumn{3}{|c|}{ Full Score } & \multicolumn{3}{|c|}{ S-M } & \multicolumn{3}{|c|}{ Supplementary S-M } \\
\hline & OR & $95 \% \mathrm{Cl}$ & $p$ Value & OR & $95 \% \mathrm{Cl}$ & $p$ Value & OR & $95 \% \mathrm{Cl}$ & $p$ Value \\
\hline Size & 1.02 & $1.00-1.05$ & 0.220 & 1.05 & $1.02-1.08$ & $<0.001$ & 1.03 & $1.00-1.06$ & 0.059 \\
\hline S-M eloquence & 0.79 & $0.34-1.86$ & 0.592 & 1.68 & $0.83-3.41$ & 0.162 & 2.13 & $1.00-4.51$ & 0.049 \\
\hline Deep venous drainage & 3.34 & $1.15-9.81$ & 0.027 & 5.26 & $1.89-13.78$ & 0.001 & 4.59 & $1.63-12.92$ & 0.004 \\
\hline Hemorrhage & 0.26 & $0.08-0.84$ & 0.024 & & & & 0.26 & $0.08-0.85$ & 0.025 \\
\hline Diffuseness & 2.22 & $0.90-5.48$ & 0.083 & & & & 2.12 & $0.89-5.05$ & 0.092 \\
\hline LED (mm) & 0.87 & $0.80-0.95$ & 0.001 & & & & & & \\
\hline Age & & & & & & & 1.02 & $1.00-1.05$ & 0.247 \\
\hline Log-transformed follow-up & 0.79 & $0.23-2.78$ & 0.716 & 0.68 & $0.22-2.06$ & 0.491 & 0.69 & $0.21-2.25$ & 0.538 \\
\hline AUC & 0.80 & $0.74-0.87$ & & 0.71 & $0.63-0.79$ & & 0.76 & $0.69-0.84$ & \\
\hline
\end{tabular}

Boldface type indicates statistical significance. 
TABLE 6. Multivariable logistic regression analysis for $\mathrm{fS}-\mathrm{M}$ grade and HDVL grade

\begin{tabular}{|c|c|c|c|c|c|c|}
\hline \multirow[b]{2}{*}{ Variables } & \multicolumn{3}{|c|}{ fS-M } & \multicolumn{3}{|c|}{ HDVL } \\
\hline & OR & $95 \% \mathrm{Cl}$ & $p$ Value & OR & $95 \% \mathrm{Cl}$ & $\mathrm{p}$ Value \\
\hline Size & 1.04 & $1.01-1.07$ & 0.004 & & & \\
\hline \multicolumn{7}{|l|}{ S-M eloquence } \\
\hline Eloquence* $^{*}$ & 6.84 & $2.50-18.71$ & $<0.001$ & & & \\
\hline Deep venous drainage & 4.28 & $1.50-12.23$ & 0.007 & 3.07 & $1.10-8.59$ & 0.033 \\
\hline Hemorrhage & & & & 0.23 & $0.07-0.74$ & 0.013 \\
\hline Diffuseness & & & & 3.00 & $1.40-6.44$ & 0.005 \\
\hline LED (mm) & & & & 0.87 & $0.81-0.94$ & $<0.001$ \\
\hline Log-transformed follow-up & 0.69 & $0.21-2.23$ & 0.535 & 0.74 & $0.24-2.79$ & 0.737 \\
\hline AUC & 0.79 & $0.72-0.86$ & & 0.82 & $0.76-0.88$ & \\
\hline
\end{tabular}

Boldface type indicates statistical significance.

* S-M eloquence plus eloquent fiber tracts.

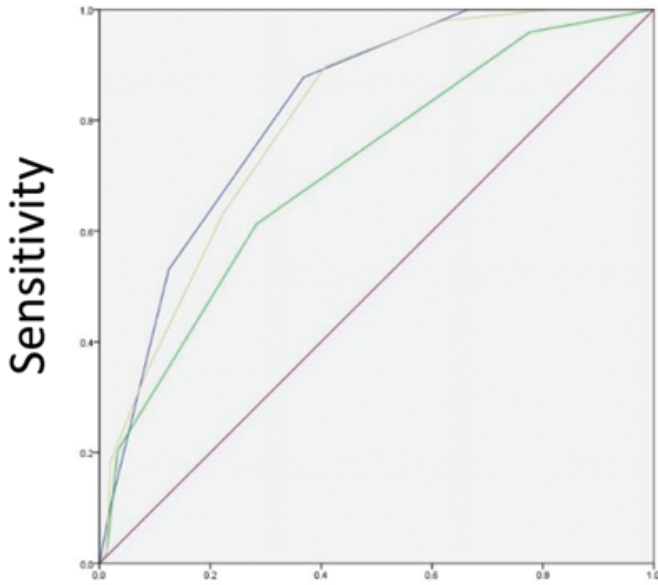

A

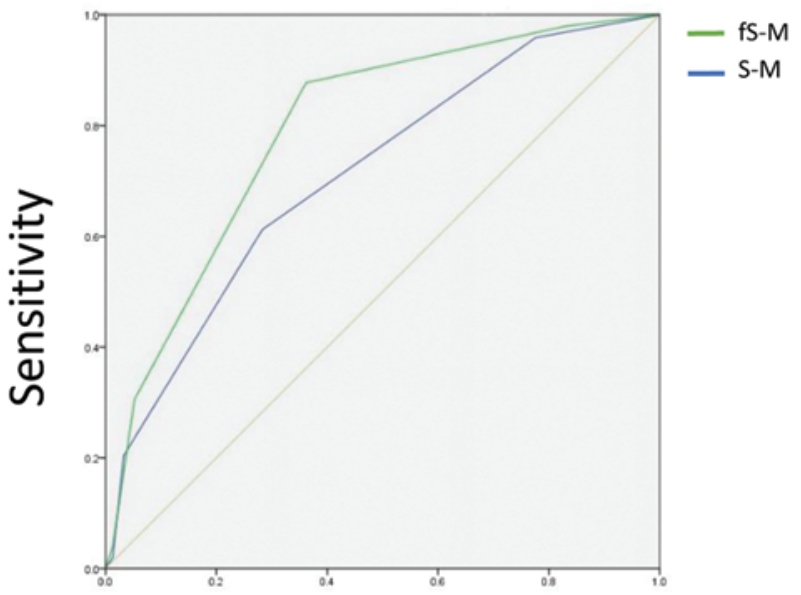

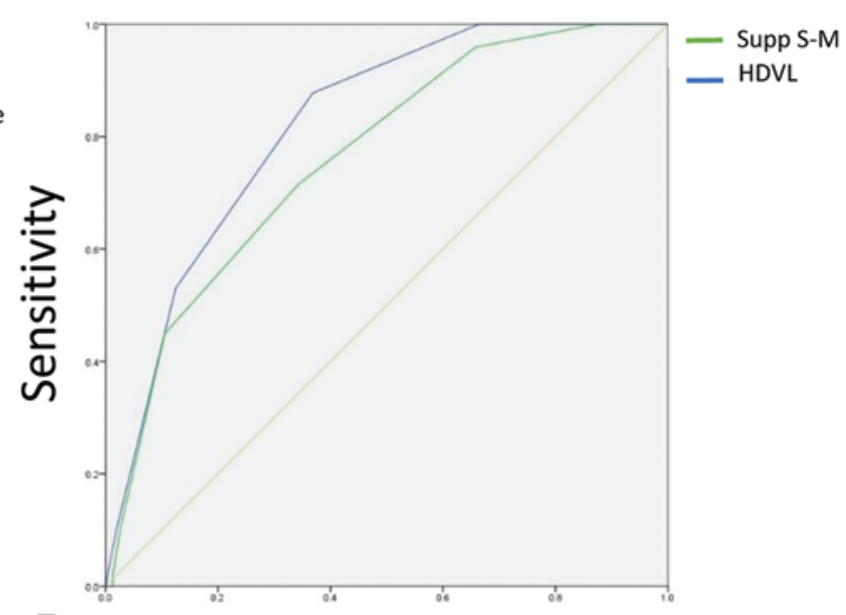

B

\section{1-Specificity}

C

\section{1-Specificity}

FIG. 4. ROC analyses for different predictive grading systems. The HDVL grading system had the highest predictive accuracy. A: ROC analysis for the HDVL grading system (blue curve), the S-M grading system (green curve), and the Full Score grading system (gray curve). The predictive accuracy of HDVL (AUC $=0.82$ ) was higher than the Full Score (AUC $=0.80$ ) and S-M (AUC $=0.71)$ grading systems. B: ROC analysis for the HDVL grading system (blue curve) and the supplementary S-M grading system (Supp S-M, green curve). The predictive accuracy of the HDVL grading system was higher than that of the supplementary S-M $(A U C=0.76)$ grading system. C: ROC analysis for the fS-M grading system (green curve) and the S-M grading system (blue curve). The predictive accuracy of the fS-M grading system (AUC $=0.79$ ) was higher than the S-M grading system. Figure is available in color online only. 
TABLE 7. Risk stratification of neurological outcomes in the derivation cohort

\begin{tabular}{|c|c|c|}
\hline \multirow[b]{2}{*}{ Grading System } & \multicolumn{2}{|c|}{ No. of Patients (\%) } \\
\hline & Improved or Unchanged & Worse \\
\hline \multicolumn{3}{|l|}{ S-M grade* } \\
\hline 1 & $10(100.0)$ & $0(0.0)$ \\
\hline 2 & $41(100.0)$ & $0(0.0)$ \\
\hline 3 & $45(88.2)$ & $6(11.8)$ \\
\hline 4 & $37(68.5)$ & $17(31.5)$ \\
\hline 5 & $16(43.2)$ & $21(56.8)$ \\
\hline 6 & $3(37.5)$ & $5(62.5)$ \\
\hline \multicolumn{3}{|l|}{ fS-M grade } \\
\hline 1 & $26(96.3)$ & $1(3.7)$ \\
\hline 2 & $71(93.4)$ & $5(6.6)$ \\
\hline 3 & $47(62.7)$ & $28(37.3)$ \\
\hline 4 & $6(31.6)$ & $13(68.4)$ \\
\hline 5 & $2(50)$ & $2(50)$ \\
\hline \multicolumn{3}{|l|}{ Full Score } \\
\hline 1 & $0(0.0)$ & $0(0.0)$ \\
\hline 2 & $6(100.0)$ & $0(0.0)$ \\
\hline 3 & $22(100.0)$ & $0(0.0)$ \\
\hline 4 & $30(96.8)$ & $1(3.2)$ \\
\hline 5 & $32(88.9)$ & $4(11.1)$ \\
\hline 6 & $28(68.3)$ & $13(31.7)$ \\
\hline 7 & $21(60.0)$ & $14(40.0)$ \\
\hline 8 & $10(55.6)$ & $8(44.4)$ \\
\hline 9 & $1(11.1)$ & $8(88.9)$ \\
\hline 10 & $2(66.7)$ & $1(33.3)$ \\
\hline \multicolumn{3}{|l|}{ HDVL grade } \\
\hline 1 & $10(100.0)$ & $0(0.0)$ \\
\hline 2 & $41(100.0)$ & $0(0.0)$ \\
\hline 3 & $45(88.2)$ & $6(11.8)$ \\
\hline 4 & 37 (68.5) & $17(31.5)$ \\
\hline 5 & $16(43.2)$ & $21(56.8)$ \\
\hline 6 & $3(37.5)$ & $5(62.5)$ \\
\hline Total & $152(75.6)$ & 49 (24.4) \\
\hline
\end{tabular}

* Arabic numerals, rather than Roman numerals, are used for the S-M grade in this table for clarity, because we have used Arabic numerals in our grading systems.

retract and bleed into the parenchyma or ventricle when disrupted.22

Both the univariate and multivariate analyses indicated that LED was another factor that was significantly correlated with surgical outcomes. Different from the resection of brain tumors, radical resection of the lesion in patients with BAVM is often necessary for intraoperative hemostasis and to reduce the risk of postoperative rebleeding. ${ }^{11}$ When the eloquent brain areas (eloquent cortex or fiber tracts) are adjacent to the nidus, particularly in patients with a diffuse BAVM nidus, rough nidus borders and intermixed adjacent brain tissue can lead to unavoidable damage by the neurosurgeon when resecting the nidus.
TABLE 8. Sensitivity, 1 - specificity, Youden Index of the HDVL grade for neurological outcomes

\begin{tabular}{cccc}
\hline HDVL & \multicolumn{3}{c}{ Diagnosis Evaluation } \\
\cline { 2 - 4 } Grade & Sensitivity & 1 - Specificity & Youden Index \\
\hline$\geq 1-6$ & 1.000 & 1.000 & 0.000 \\
\hline$\geq 2-6$ & 1.000 & 0.934 & 0.066 \\
\hline$\geq 3-6$ & 1.000 & 0.664 & 0.336 \\
\hline$\geq 4-6$ & 0.878 & 0.368 & 0.509 \\
\hline$\geq 5-6$ & 0.531 & 0.125 & 0.406 \\
\hline 6 & 0.102 & 0.020 & 0.082 \\
\hline
\end{tabular}

Therefore, the relationship between nidus and eloquent brain areas appears to be crucial in the preoperative evaluation of BAVM. Generally, the adjacent eloquent brain was mostly identified by anatomy from the traditional MRI study. ${ }^{15,22}$ However, structural anatomy defined by the traditional MRI as eloquent does not always equate with functional anatomy, because a brain harboring a BAVM often relocates functional areas that lie too close to the nidus. ${ }^{2,3,14,19}$ Preoperative imaging with fMRI and DTI tractography have helped us more precisely localizes a patient's eloquent cortex and white matter functional fiber tracts. Moreover, the relationship between the nidus and eloquent brain areas can be quantified by the LED with the aid of BOLD fMRI and DTI tractography. Thus, LED was of great significance in predicting the surgical outcomes in patients with BAVMs. The ROC analyses showed that an LED of $4.95 \mathrm{~mm}$ was the cutoff point for the worsened mRS score. Therefore, according to our data, an LED less than $5 \mathrm{~mm}(4.95-5 \mathrm{~mm})$ could be regarded as eloquent adjacent brain.

Our data showed that the predictive value of the HDVL grading system was better than that of the S-M and the supplementary S-M grading systems. The S-M grading system is the most popular prediction model. In our study, a high S-M grade was significantly associated with a worsened mRS score. This result was consistent with those of previous studies. ${ }^{9}$ However, a previous study showed that the elements of the S-M grading system were not independent. With increasing AVM size, an eloquent brain location and a deep venous drainage component became more frequent. The correlation within the elements of the S-M grading system weakened its predictive value..$^{10}$ Similarly, in our study, multivariate analysis revealed that only the nidus size and deep venous drainage of the S-M grading system were independent predictors. In addition, when the variables were tested along with the other predictors (LED, diffuseness, and nonhemorrhagic presentation) using multivariate analysis, only deep venous drainage in the S-M grading system was an independent predictor for surgical morbidity. It is possible that the significance of the size and eloquence were accounted for indirectly by the other predictors, especially the LED. In this study, patient age was not correlated with surgical outcomes. This was a significant reason for the low predictive value of the supplementary S-M grading system. In contrast, in the HDVL grading system, the included elements were all independent 
predictors for surgical morbidity. Moreover, different from the S-M grading system or the supplementary S-M grading system, the HDVL grading scale's predictive accuracy is improved by adding information regarding the eloquent fiber tracts and quantification of the relationship between the nidus and eloquent cortex and fiber tracts effectively.

According to our data, the HDVL model had the highest predictive accuracy. The fS-M model also had a higher predictive accuracy than the S-M grading system. Both systems demonstrated the importance of the eloquent fiber tracts in the preoperative evaluation of the surgical outcomes. We envisioned that the HDVL and fS-M grading systems would assist rather than replace the already well-established S-M grading system. If fMRI and DTI were available, an analysis of the HDVL and fS-M grading systems could impact a management decision with a higher predictive accuracy. An AVM with a low S-M grade (Grades I-III) and a low HDVL grade (Grades 1-3) may strengthen the recommendation for surgery (Fig. 2). Conversely, a BAVM with a high S-M grade (Grade IV or V) and a high HDVL grade (Grades 4-6) may strengthen the recommendation for nonoperative management. In cases of mismatched S-M and HDVL grades, the HDVL grading system may have a more important role and alter clinical decisions that are made using the S-M grading system (Figs. 1 and 3). Evaluation without fMRI or DTI may reduce the predictive value.

\section{Limitations}

There are limitations to our study. Conclusions are limited due to our retrospective approach; it was difficult to avoid selection bias and confounding factors. In addition, patients with supratentorial BAVMs were enrolled in our database. Furthermore, external validation of the score is missing. Further validation with a prospective approach and more patients, including both supra- and infratentorial BAVMs, should be performed.

\section{Conclusions}

In this study, LED was a significant predictor for the preoperative risk evaluation for surgery. The HDVL system is a good predictive grading scale of neurological outcomes after BAVM surgery. Adding information regarding eloquent fiber tracts to preoperative evaluation can effectively improve its predictive accuracy.

\section{Acknowledgments}

This study was supported by the "Key Projects in the National Science \& Technology Pillar Program during the Twelfth Five-Year Plan Period" (Grant No. 2011BAI08B08, Principal Investigator: Professor Shuo Wang) and the "Key Project of Beijing Municipal Science \& Technology Commission" (Grant No. D161100003816006, Principal Investigator: Professor Shuo Wang). In addition, this study was supported by the "973 National Key Basic Research Development Plan" (Grant No. 2012CB720704, Principal Investigator: Professor Zhen Jin).

\section{References}

1. Abd-El-Barr MM, Saleh E, Huang RY, Golby AJ: Effect of disease and recovery on functional anatomy in brain tumor patients: insights from functional MRI and diffusion tensor imaging. Imaging Med 5:333-346, 2013

2. Alkadhi H, Kollias SS, Crelier GR, Golay X, Hepp-Reymond MC, Valavanis A: Plasticity of the human motor cortex in patients with arteriovenous malformations: a functional MR imaging study. AJNR Am J Neuroradiol 21:1423-1433, 2000

3. Baciu M, Le Bas JF, Segebarth C, Benabid AL: Presurgical fMRI evaluation of cerebral reorganization and motor deficit in patients with tumors and vascular malformations. Eur $\mathbf{J}$ Radiol 46:139-146, 2003

4. Bendok BR, El Tecle NE, El Ahmadieh TY, Koht A, Gallagher TA, Carroll TJ, et al: Advances and innovations in brain arteriovenous malformation surgery. Neurosurgery 74 (Suppl 1):S60-S73, 2014

5. Buxton RB: The physics of functional magnetic resonance imaging (fMRI). Rep Prog Phys 76:096601, 2013

6. Du R, Dowd CF, Johnston SC, Young WL, Lawton MT: Interobserver variability in grading of brain arteriovenous malformations using the Spetzler-Martin system. Neurosurgery 57:668-675, 2005

7. Du R, Keyoung HM, Dowd CF, Young WL, Lawton MT: The effects of diffuseness and deep perforating artery supply on outcomes after microsurgical resection of brain arteriovenous malformations. Neurosurgery 60:638-648, 2007

8. Ellis MJ, Rutka JT, Kulkarni AV, Dirks PB, Widjaja E: Corticospinal tract mapping in children with ruptured arteriovenous malformations using functionally guided diffusiontensor imaging. J Neurosurg Pediatr 9:505-510, 2012

9. Hamilton MG, Spetzler RF: The prospective application of a grading system for arteriovenous malformations. Neurosurgery 34:2-7, 1994

10. Hartmann A, Stapf C, Hofmeister C, Mohr JP, Sciacca RR, Stein BM, et al: Determinants of neurological outcome after surgery for brain arteriovenous malformation. Stroke 31:2361-2364, 2000

11. Hashimoto N, Nozaki K, Takagi Y, Kikuta K, Mikuni $\mathrm{N}$ : Surgery of cerebral arteriovenous malformations. Neurosurgery 61 (1 Suppl):375-389, 2007

12. Hofmeister C, Stapf C, Hartmann A, Sciacca RR, Mansmann $\mathrm{U}$, terBrugge $\mathrm{K}$, et al: Demographic, morphological, and clinical characteristics of 1289 patients with brain arteriovenous malformation. Stroke 31:1307-1310, 2000

13. Hong JH, Kim SH, Ahn SH, Jang SH: The anatomical location of the arcuate fasciculus in the human brain: a diffusion tensor tractography study. Brain Res Bull 80:5255,2009

14. Jang SH, Hong JH, Ahn SH, Son SM, Cho YW: Motor function reorganization in a patient with a brainstem lesion: DTT, fMRI and TMS study. NeuroRehabilitation 26:167171,2010

15. Lawton MT, Kim H, McCulloch CE, Mikhak B, Young WL: A supplementary grading scale for selecting patients with brain arteriovenous malformations for surgery. Neurosurgery 66:702-713, 2010

16. Lepski G, Honegger J, Liebsch M, Sória MG, Narischat P, Ramina KF, et al: Safe resection of arteriovenous malformations in eloquent motor areas aided by functional imaging and intraoperative monitoring. Neurosurgery 70 (2 Suppl Operative):276-289, 2012

17. Lin F, Zhao B, Wu J, Wang L, Jin Z, Cao Y, et al: Risk factors for worsened muscle strength after the surgical treatment of arteriovenous malformations of the eloquent motor area. $\mathbf{J}$ Neurosurg 125:289-298, 2015

18. Pillai JJ: The evolution of clinical functional imaging during the past 2 decades and its current impact on neurosurgical planning. AJNR Am J Neuroradiol 31:219-225, 2010

19. Scantlebury N, Gaetz W, Widjaja E, Rutka J, Bouffet E, 
Rockel C, et al: Functional reorganization of the corticospinal tract in a pediatric patient with an arteriovenous malformation. Neuroreport 25:55-59, 2014

20. Sherbondy AJ, Dougherty RF, Napel S, Wandell BA: Identifying the human optic radiation using diffusion imaging and fiber tractography. J Vis 8:12.1-12.11, 2008

21. Spears J, TerBrugge KG, Moosavian M, Montanera W, Willinsky RA, Wallace MC, et al: A discriminative prediction model of neurological outcome for patients undergoing surgery of brain arteriovenous malformations. Stroke 37:1457-1464, 2006

22. Spetzler RF, Martin NA: A proposed grading system for arteriovenous malformations. 1986. J Neurosurg 108:186193,2008

23. Tong X, Wu J, Lin F, Cao Y, Zhao Y, Jin Z, et al: Visual field preservation in surgery of occipital arteriovenous malformations: a prospective study. World Neurosurg 84:1423-1436, 2015

24. Wang S, Poptani H, Bilello M, Wu X, Woo JH, Elman LB, et al: Diffusion tensor imaging in amyotrophic lateral sclerosis: volumetric analysis of the corticospinal tract. AJNR Am J Neuroradiol 27:1234-1238, 2006

25. Zhao B, Cao Y, Zhao Y, Wu J, Wang S: Functional MRI-guided microsurgery of intracranial arteriovenous malformations: study protocol for a randomised controlled trial. BMJ Open 4:e06618, 2014

\section{Disclosures}

The authors report no conflict of interest concerning the materials or methods used in this study or the findings specified in this paper.

\section{Author Contributions}

Conception and design: Cao, Jiao, Lin. Acquisition of data: Jiao, Lin, Wu, Li, L Wang, Jin, S Wang. Analysis and interpretation of data: Cao, Jiao, Lin. Drafting the article: Jiao. Critically revising the article: Cao, Jiao. Reviewed submitted version of manuscript: Cao, Jiao, Lin, Li, S Wang. Statistical analysis: Jiao, Wu, Li. Administrative/technical/material support: Lin, Wu, Li, L Wang, Jin, S Wang. Study supervision: Cao, Jin, S Wang.

\section{Correspondence}

Yong Cao, Department of Neurosurgery, Beijing Tiantan Hospital, Capital Medical University, No. 6 Tiantanxili, Dongcheng district, Beijing 100050, China. email: caolaoban6@163.com. 